\title{
RESEARCH
}

Open Access

\section{Factors influencing liberation from mechanical ventilation in coronavirus disease 2019: multicenter observational study in fifteen Italian ICUs}

Lorenzo Gamberini ${ }^{1}$, Tommaso Tonetti ${ }^{2 *}$ (D), Savino Spadaro ${ }^{3}$, Gianluca Zani ${ }^{4}$, Carlo Alberto Mazzoli ${ }^{1}$, Chiara Capozzi ${ }^{5}$, Emanuela Giampalma ${ }^{6}$, Maria Letizia Bacchi Reggiani ${ }^{7}$, Elisabetta Bertellini ${ }^{8}$, Andrea Castelli ${ }^{5}$, Irene Cavalli ${ }^{2}$, Davide Colombo ${ }^{9,10}$, Federico Crimaldi ${ }^{11}$, Federica Damiani ${ }^{12}$, Alberto Fogagnolo ${ }^{3}$, Maurizio Fusari ${ }^{4}$, Emiliano Gamberini ${ }^{13}$, Giovanni Gordini ${ }^{1}$, Cristiana Laici ${ }^{14}$, Maria Concetta Lanza ${ }^{15}$, Mirco Leo ${ }^{16}$, Andrea Marudi ${ }^{8}$, Giuseppe Nardi ${ }^{17}$, Irene Ottaviani ${ }^{3}$, Raffaella Papa ${ }^{18}$, Antonella Potalivo ${ }^{17}$, Emanuele Russo ${ }^{13}$, Stefania Taddei ${ }^{19}$, Carlo Alberto Volta ${ }^{3}$, V. Marco Ranieri ${ }^{2}$ and the ICU-RER COVID-19 Collaboration

\begin{abstract}
Background: A large proportion of patients with coronavirus disease 2019 (COVID-19) develop severe respiratory failure requiring admission to the intensive care unit (ICU) and about $80 \%$ of them need mechanical ventilation (MV). These patients show great complexity due to multiple organ involvement and a dynamic evolution over time; moreover, few information is available about the risk factors that may contribute to increase the time course of mechanical ventilation. The primary objective of this study is to investigate the risk factors associated with the inability to liberate COVID-19 patients from mechanical ventilation. Due to the complex evolution of the disease, we analyzed both pulmonary variables and occurrence of non-pulmonary complications during mechanical ventilation. The secondary objective of this study was the evaluation of risk factors for ICU mortality.

Methods: This multicenter prospective observational study enrolled 391 patients from fifteen COVID-19 dedicated Italian ICUs which underwent invasive mechanical ventilation for COVID-19 pneumonia. Clinical and laboratory data, ventilator parameters, occurrence of organ dysfunction, and outcome were recorded. The primary outcome measure was 28 days ventilator-free days and the liberation from MV at 28 days was studied by performing a competing risks regression model on data, according to the method of Fine and Gray; the event death was considered as a competing risk.

(Continued on next page)
\end{abstract}

\footnotetext{
*Correspondence: tommaso.tonetti@unibo.it

${ }^{2}$ Alma Mater Studiorum, Dipartimento di Scienze Mediche e Chirurgiche,

Anesthesia and Intensive Care Medicine, Policlinico di Sant'Orsola, Università di Bologna, Bologna, Italy

Full list of author information is available at the end of the article
}

(c) The Author(s). 2020, corrected publication 2020. Open Access This article is licensed under a Creative Commons Attribution 4.0 International License, which permits use, sharing, adaptation, distribution and reproduction in any medium or format, as long as you give appropriate credit to the original author(s) and the source, provide a link to the Creative Commons licence, and indicate if changes were made. The images or other third party material in this article are included in the article's Creative Commons licence, unless indicated otherwise in a credit line to the material. If material is not included in the article's Creative Commons licence and your intended use is not permitted by statutory regulation or exceeds the permitted use, you will need to obtain permission directly from the copyright holder. To view a copy of this licence, visit http://creativecommons.org/ licenses/by/4.0/. The Creative Commons Public Domain Dedication waiver (http://creativecommons.org/publicdomain/zero/1. 0/) applies to the data made available in this article, unless otherwise stated in a credit line to the data. 
(Continued from previous page)

Results: Liberation from mechanical ventilation was achieved in 53.2\% of the patients (208/391). Competing risks analysis, considering death as a competing event, demonstrated a decreased sub-hazard ratio for liberation from mechanical ventilation (MV) with increasing age and SOFA score at ICU admission, low values of $\mathrm{PaO}_{2} / \mathrm{FiO}_{2}$ ratio during the first 5 days of $\mathrm{MV}$, respiratory system compliance $\left(\mathrm{C}_{\mathrm{RS}}\right)$ lower than $40 \mathrm{~mL} / \mathrm{cmH}_{2} \mathrm{O}$ during the first 5 days of $\mathrm{MV}$, need for renal replacement therapy (RRT), late-onset ventilator-associated pneumonia (VAP), and cardiovascular complications. ICU mortality during the observation period was $36.1 \%$ (141/391). Similar results were obtained by the multivariate logistic regression analysis using mortality as a dependent variable.

Conclusions: Age, SOFA score at ICU admission, $\mathrm{C}_{\mathrm{RS}}, \mathrm{PaO}_{2} / \mathrm{FiO}_{2}$, renal and cardiovascular complications, and late-onset VAP were all independent risk factors for prolonged mechanical ventilation in patients with COVID-19.

Trial registration: NCT04411459

Keywords: Coronavirus disease 2019, Intensive care, Mechanical ventilation, Outcomes, mortality, Respiration, artificial, ARDS

\section{Introduction}

A large proportion of patients with coronavirus disease 2019 (COVID-19) develop severe respiratory failure requiring admission to the intensive care unit (ICU) and about $80 \%$ of them need mechanical ventilation (MV) [1].

The reported mortality rate of mechanically ventilated patients with COVID-19 ranges from 30 to 97\% [2-6]. Such a large variability may-at least in part-be due to the fact that a large portion of patients was still on mechanical ventilation in most of these studies. It is therefore relevant to identify the risk factors associated with a longer duration of mechanical ventilation. Of note, COVID-19 patients show great complexity due to multiple organ involvement, such as the lung, heart, kidney, and nervous system [7]. All these clinical features can contribute to the inability to liberate patients from MV. This may be particularly relevant since the majority of studies to date are focused mostly on admission clinical variables. Therefore, since COVID-19 is characterized by a dynamic evolution over time [8], few information is available on the risk factors that may contribute to increase the time course of mechanical ventilation, delaying the recovery to spontaneous ventilation.

The primary objective of this study is to investigate the risk factors associated with the inability to liberate COVID-19 patients from mechanical ventilation. Due to the complex evolution of the disease, we analyzed both pulmonary variables and the occurrence of nonpulmonary complications during mechanical ventilation.

\section{Methods}

We conducted a prospective multicenter observational study in 15 ICUs. All consecutive patients with laboratory-confirmed SARS-CoV-2 infection admitted to participating dedicated COVID-19 ICUs from the 22nd of February through the 4th of May (the end of the nation-wide lockdown in Italy) 2020 were screened for eligibility. The clinical outcomes were monitored up to May 15, the final date of follow-up.

\section{Inclusion and exclusion criteria}

Patients older than 18 years who received invasive mechanical ventilation were considered as eligible. Inclusion criteria were (a) SARS-CoV-2 infection confirmed by realtime reverse transcription-polymerase chain reaction assays from either nasal swabs or lower respiratory tract samples [9] and (b) use of invasive mechanical ventilation at any time of the clinical course. Exclusion criteria were (a) not a laboratory-confirmed SARS-CoV-2, (b) use of extracorporeal membrane oxygenation (ECMO) and/or extracorporeal $\mathrm{CO}_{2}$ removal $\left(\mathrm{ECCO}_{2} \mathrm{R}\right)$, (c) use of noninvasive ventilation during the entire clinical course, and (d) cardiac arrest before intubation.

Patients with missing data for the variables of interest (comorbidities, length of mechanical ventilation, ventilatory parameters during the first 5 days, ICU complications, and discharge information) were not included in the final analysis.

The study was approved by the Institutional Review Board (IRB) of the study coordinator center (Maggiore Hospital, Bologna, Italy, approval number: 273/2020/OSS/AUSLBO) and by each institutional review committee of the participating hospitals. Informed consent was partially waived according to the approval of the local ethics committee, and researchers analyzed anonymized individual data. The study was registered in ClinicalTrials.gov (NCT04411459).

\section{Data collection}

A list of clinical variables was defined before the initiation of the study using a priori designed case report forms. Data were collected by one investigator of each hospital in an electronic case report form developed by YGHEA, CRO division of Ecol Studio SPA (Bologna Operational Headquarters), and hosted by ACTide Nubilaria (Novara, Italy). Collected data comprised demographic data, information on clinical symptoms, or signs at presentation, underlying comorbidities, laboratory findings, imaging studies, and respiratory parameters before the intubation and ventilator setting during the first 5 days of mechanical 
ventilation (e.g., positive end-expiratory pressure (PEEP), plateau pressure (Pplat), static compliance $\left(\mathrm{C}_{\mathrm{RS}}\right), \mathrm{PaO}_{2} /$ $\mathrm{FiO}_{2}$ ratio), and clinical outcomes. Additional details on collected variables are available in the online supplement.

\section{Outcome variables}

Ventilator-free days at day 28 was the primary outcome variable. Risk factors associated with ICU mortality were also assessed.

\section{Definitions}

Mechanical ventilation was considered invasive if delivered through an endotracheal tube or a tracheostomy. The duration of mechanical ventilation was defined as the time elapsed from intubation to extubation or successful disconnection from mechanical ventilation for tracheostomized patients. Extubation failure was defined as the need for reintubation within $48 \mathrm{~h}$, and the time from failed extubation to reintubation was recorded.

ARDS was classified into three increasing levels of oxygenation failure into mild, moderate, and severe, according to the Berlin definition of ARDS [10].

For respiratory system compliance, we chose a cut-off of $40 \mathrm{ml} / \mathrm{cmH}_{2} \mathrm{O}$ for discriminating between higher and lower compliance. This cut-off was previously proposed in the Berlin definition of ARDS as an ancillary variable for defining the most severe cases [10].

Ventilator-free days (VFDs) were defined as a time frame of 28 days from intubation. For intubated patients, in case of reintubation within 28 days, VFDs were counted from the last successful extubation. The use of non-invasive ventilation (NIV) after extubation was not considered as a ventilation period. Finally, zero VFDs were assigned to 28-day non-survivors, regardless of their intubation status [11]. In tracheostomized patients, interval disconnections were not counted and VFDs started after the last successful disconnection from mechanical ventilation [11].

\section{Statistical analysis}

Data were analyzed using Stata/SE 15.1 (College Station, Texas, USA); continuous variables were expressed as the median and interquartile range (IQR); comparisons between continuous variables were performed with Mann-Whitney $U$ test; categorical variables were expressed as numbers and percentages and compared using chi-squared test.

The liberation from MV at 28 days was studied by performing a competing risks regression model on data, according to the method of Fine and Gray; the event death was considered as a competing risk [11].

Model building was performed by means of a variable selection based on an initial screening using univariate analysis with $p<0.2$ criteria, then a stepwise selection with entry criteria $=0.05$ and stay criteria $=0.1$.
Estimates of coefficients in the model are reported as sub-hazard ratios along with the graphs of the cumulative incidence function of liberation from $\mathrm{MV}$ on the basis of either static compliance of the respiratory system or $\mathrm{PaO}_{2} / \mathrm{FiO}_{2}$ ratio range adjusted for the other covariates introduced into the multivariate model.

Univariate and multivariable logistic regression analyses were performed in order to evaluate factors associated with death during ICU stay. Screened risk variables and model building were the same as for competing risks regression. The area under the receiver operator characteristic (ROC) was reported.

In all analyses, the standard errors were adjusted considering enrolling centers as clusters; therefore, assuming that observations were independent across different hospitals but not necessarily within the same center. All $p$ values refer to two-tailed tests of significance and $p<$ 0.05 was considered significant.

\section{Results \\ Population}

Over the study period, 607 patients were screened for eligibility. Patients excluded for admission for other causes than respiratory failure, use of $\mathrm{ECMO} / \mathrm{ECCO} 2 \mathrm{R}$, use of noninvasive ventilation during the entire clinical course, and cardiac arrest before intubation were $48,11,67$, and 6 , respectively. Eighty-four patients had missing data for the variables of interest and were not included for the final analysis. Three hundred ninety-one patients were therefore included in the final analysis (figure S1, online supplement).

Main demographics, comorbidities, and clinical characteristics at ICU admission are detailed in Table 1. The patients were predominantly male $(300 / 391,77 \%)$, with a median [IQR] age of 66 years [59-72]. Hypertension was the most common comorbidity (222/391, 57\%).

Non-invasive ventilation or CPAP was applied in 254 patients $(65 \%)$ before tracheal intubation. Fifty-four of them (21\% of patients receiving NIV/CPAP) received NIV in the ICU for more than $24 \mathrm{~h}$. Patients were intubated after a median of 2 [1-5] days from hospital admission.

Before starting $\mathrm{MV}$, the median $\mathrm{PaO}_{2} / \mathrm{FiO}_{2}$ ratio was 94 [75 - 119] mmHg. Initial respiratory parameters showed a median positive end-expiratory pressure (PEEP) of 12 [10 - 14] $\mathrm{cmH}_{2} \mathrm{O}$ which resulted in a static respiratory system compliance $\left(\mathrm{C}_{\mathrm{RS}}\right)$ of $38 \mathrm{~mL} / \mathrm{cmH}_{2} \mathrm{O}$ [31 - 47]. During the first 5 days of mechanical ventilation, the lowest recorded $\mathrm{PaO}_{2} / \mathrm{FiO}_{2}$ ratio was 100 [76-132]. Almost all patients (96.2\%) had a $\mathrm{PaO}_{2} / \mathrm{FiO}_{2}$ ratio lower than 200. Tracheostomy was performed in 224 patients $(57.3 \%)$ with a median time from orotracheal intubation to tracheostomy of 9 [5 - 12] days. Two-hundred fifty-eight patients (66.0\%) were treated with at least one cycle of prone positioning and 365 patients $(93 \%)$ received a continuous infusion of neuromuscular blocking agents (NMBA) for at least $24 \mathrm{~h}$ 
Table 1 Main patients characteristics

\begin{tabular}{|c|c|c|c|c|}
\hline & $\begin{array}{l}\text { Total population } \\
(n=391)\end{array}$ & $\begin{array}{l}\text { Successful liberation from } \\
\text { MV } 28 \text { days }(n=165)\end{array}$ & $\begin{array}{l}\text { Unsuccessful liberation from } \\
\text { MV } 28 \text { days }(n=226)\end{array}$ & $p$ \\
\hline \multicolumn{5}{|l|}{ Variables } \\
\hline Age - year (IQR) & $66(59-72)$ & $64(56-70)$ & $68(62-74)$ & $<0.001$ \\
\hline Sex - male - no (\%) & $300(76.7 \%)$ & $121(73.3 \%)$ & 179 (79.2\%) & 0.175 \\
\hline BMI - median (IQR) & $28(26-31)$ & $28(26-31)$ & $28(26-31)$ & 0.918 \\
\hline SAPS II score & $38(31-46)$ & $35(28-42)$ & $39(33-48)$ & $<0.001$ \\
\hline SOFA score at ICU admission & $5(3-7)$ & $4(3-6)$ & $6(4-8)$ & $<0.001$ \\
\hline \multicolumn{5}{|l|}{ Comorbidities } \\
\hline Hypertension - no (\%) & $222(56.8 \%)$ & $79(47.9 \%)$ & $143(63.3 \%)$ & 0.003 \\
\hline Chronic ischemic heart disease - no (\%) & $35(9.0 \%)$ & $12(7.3 \%)$ & $23(10.2 \%)$ & 0.416 \\
\hline Chronic kidney disease (CKD) & $26(6.6 \%)$ & $8(4.8 \%)$ & $18(8.0 \%)$ & 0.362 \\
\hline CKD - patients in dialisys - no (\%) & $4(1.0 \%)$ & $1(0.6 \%)$ & $3(1.3 \%)$ & \\
\hline COPD - no (\%) & $27(6.9 \%)$ & $11(6.7 \%)$ & $16(7.1 \%)$ & $0.964^{a}$ \\
\hline COPD - home oxygen therapy/CPAP - no (\%) & $2(0.5 \%)$ & $1(0.6 \%)$ & $1(0.4 \%)$ & \\
\hline Diabetes - no (\%) & $85(21.7 \%)$ & $28(17.0 \%)$ & $57(25.2 \%)$ & 0.067 \\
\hline Chronic liver disease (MELD > 10) - no (\%) & $3(0.8 \%)$ & $1(0.6 \%)$ & $2(0.9 \%)$ & 1.000 \\
\hline Active cancer - no (\%) & $7(1.8 \%)$ & $0(0 \%)$ & $7(3.1 \%)$ & 0.058 \\
\hline Immunosuppressive therapy - no (\%) & $10(2.6 \%)$ & $1(0.6 \%)$ & $9(4.0 \%)$ & 0.078 \\
\hline \multicolumn{5}{|l|}{ Smoker status $(n=213)^{b}$} \\
\hline Current - no (\%) & $13(6.1 \%)$ & $8(8.1 \%)$ & $5(4.4 \%)$ & $0.507^{a}$ \\
\hline Previous - no (\%) & $66(31.0 \%)$ & $29(29.3 \%)$ & $37(32.5 \%)$ & \\
\hline \multicolumn{5}{|l|}{ Characteristics before ICU admission } \\
\hline $\begin{array}{l}\text { Time from symptoms onset to hospital admission } \\
(n=327)^{b}-d \text { (IQR) }\end{array}$ & $7(4-9)$ & $7(4-9)$ & $7(4-10)$ & 0.480 \\
\hline Time from hospital admission to ICU admission - d (IQR) & $2(1-5)$ & $2(1-5)$ & $2(0-5)$ & 0.436 \\
\hline Time from hospital admission to intubation - $d$ (IQR) & $2(1-5)$ & $2(1-5)$ & $2(1-6)$ & 0.389 \\
\hline \multicolumn{5}{|l|}{ Ward of admission } \\
\hline Emergency department - no (\%) & $70(17.9 \%)$ & $20(12.1 \%)$ & $50(22.1 \%)$ & $0.04^{a}$ \\
\hline Medical ward - no (\%) & $184(47.1 \%)$ & $82(49.7 \%)$ & $102(45.1 \%)$ & \\
\hline Other ICU - no (\%) & $137(35.0 \%)$ & $63(38.2 \%)$ & $74(32.7 \%)$ & \\
\hline $\begin{array}{l}\text { High flow nasal oxygen therapy before intubation } \\
(n=362)^{b}-\text { no }(\%)\end{array}$ & $31(8.5 \%)$ & $14(9.1 \%)$ & $17(8.1 \%)$ & 0.880 \\
\hline $\begin{array}{l}\text { CPAP/Non-invasive ventilation trial before intubation } \\
(n=363)^{b}-\text { no }(\%)\end{array}$ & $254(70 \%)$ & $117(70.9 \%)$ & $137(60.6 \%)$ & 0.029 \\
\hline \multicolumn{5}{|l|}{ Duration of CPAP/NIV trial $(n=363)^{b}$} \\
\hline$<12 \mathrm{~h}-\mathrm{no}(\%)$ & $56(22.1 \%)$ & $24(20.5 \%)$ & $32(23.5 \%)$ & $0.051^{a}$ \\
\hline $12-24 \mathrm{~h}-\mathrm{no}(\%)$ & $63(24.9 \%)$ & $35(29.9 \%)$ & $28(20.6 \%)$ & \\
\hline $24-48$ h - no (\%) & $44(17.4 \%)$ & $25(21.4 \%)$ & $19(14.0 \%)$ & \\
\hline$>48 \mathrm{~h}-\mathrm{no}(\%)$ & $90(35.6 \%)$ & $33(28.2 \%)$ & $57(41.9 \%)$ & \\
\hline $\mathrm{PaO}_{2} / \mathrm{FiO}_{2}$ ratio before intubation $(n=297)^{\mathrm{b}}$ & $94(75-119)$ & $96(71-125)$ & $91(77-116)$ & 0.544 \\
\hline
\end{tabular}

Abbreviations: IQR interquartile range, BMI body mass index, SAPS simplified acute physiology score, SOFA Sequential Organ Failure Assessment Score, CKD chronic kidney disease, COPD chronic obstructive pulmonary disease, CPAP continuous positive airway pressure, MELD model for end-stage liver disease, $\mathrm{NIV}$ non-invasive ventilation, $\mathrm{PaO}_{2}$ arterial oxygen partial pressure, $\mathrm{FiO}_{2}$ inspired fraction of oxygen

${ }^{a} p$ value referred to Pearson's chi-square test performed on the contingency table represented

Incomplete data due to transfer from other ICUs without complete medical records 
(see Table S1, online supplement for further details about adjunctive treatments) (Table 2).

In this cohort, 216 patients $(55.2 \%)$ experienced at least one non-pulmonary complication, the most frequent being acute kidney injury needing renal replacement therapy (76 patients,19.4\%) and cardiovascular complications (66 patients, 16.9\%).

Among pulmonary complications, the most frequent was the late-onset ventilator-associated pneumonia (175 patients, 44.8\%). Table S2 (online supplement) describes the specific complications observed.

During the period of observation, liberation from MV was achieved in 208 patients (53.2\%) after a mean duration of MV of 14 [9 - 19] days. Clinical characteristics, as well as respiratory parameters and occurrences of non-pulmonary organ failure, are reported in Table 2. Initial ventilatory variables did not show differences between groups. During the first 5 days of $\mathrm{MV}$, both $\mathrm{PaO}_{2} /$ $\mathrm{FiO}_{2}$ ratio and $\mathrm{C}_{\mathrm{RS}}$ were significantly higher in patients who achieved a successful liberation.

Competing risks analysis demonstrated a decreased sub-hazard ratio (SHR) for liberation from mechanical ventilation with increasing age and SOFA score at ICU admission, with decreasing lowest $\mathrm{PaO}_{2} / \mathrm{FiO}_{2}$ ratio and $\mathrm{C}_{\mathrm{RS}}$ lower than $40 \mathrm{~mL} / \mathrm{cmH}_{2} \mathrm{O}$ during the first 5 days of $\mathrm{MV}$, need for renal replacement therapy (RRT) during ICU stay, late-onset ventilator-associated pneumonia (VAP), and cardiovascular complications. Univariate and multivariate analyses for liberation from mechanical ventilation are presented in Table 3.

Figure 1 shows the cumulative incidence function of 28-day liberation from mechanical ventilation divided for classes of worst $\mathrm{C}_{\mathrm{RS}}$ or $\mathrm{PaO}_{2} / \mathrm{FiO}_{2}$ ratio observed within the first 5 days of $\mathrm{MV}$ and adjusted for the significant covariates defined in Table 3.

\section{Secondary outcome}

Multivariate logistic regression analysis using mortality as a dependent variable showed that increasing age, higher SOFA score at ICU admission, need for renal replacement therapy during ICU stay, cardiovascular complications, lower $\mathrm{PaO}_{2} / \mathrm{FiO}_{2}$ ratio, and low $\mathrm{C}_{\mathrm{RS}}$ during the first 5 days of invasive MV were independently associated to ICU mortality. Table 4 shows the results of univariate and multivariate analyses on the abovementioned variables (see Figure S2 online supplement for the ROC curve).

\section{Discussion}

The main findings of this prospective analysis are (a) invasively ventilated COVID-19 patients exhibited both a long duration of mechanical ventilation and ICU length of stay; (b) age, SOFA score, $\mathrm{PaO}_{2} / \mathrm{FiO}_{2}$ ratio, $\mathrm{C}_{\mathrm{RS}}$, acute kidney injury (requiring renal replacement therapy), late pulmonary infections, and cardiovascular complications were all independent risk factors for prolonged mechanical ventilation; and (c) our patients' population had $\mathrm{PaO}_{2} / \mathrm{FiO}_{2}$ and $\mathrm{C}_{\mathrm{RS}}$ values resembling those of "classical" (i.e., non-COVID-19 related) ARDS.

Most observational studies published until now on COVID-19 patients investigated the risk factors for hospital mortality, whereas our aim was to focus on risk factors responsible for prolonged mechanical ventilation.

The risk factors we identified may be classified into three main categories: demographics and baseline severity, physiology, and complications during the ICU stay.

Among demographical variables, increasing age was significantly associated with a higher duration of MV and ICU mortality and this is in line with recent literature on COVID-19 patients [12], but also on "classical" ARDS patients $[2,13]$. As for baseline severity, SOFA score at ICU admission, but not SAPS II score, was inversely associated to an increased SHR for liberation from mechanical ventilation at 28 days and directly associated to death in the multivariate competing risk and logistic analyses $[2,14,15]$.

Considering the physiological characteristics of the patients, most of them exhibited a reduced static compliance. A $\mathrm{C}_{\mathrm{RS}}<40 \mathrm{~mL} / \mathrm{cmH}_{2} \mathrm{O}$ was previously proposed in the Berlin definition of ARDS as an ancillary variable for defining the most severe cases [10]; moreover, this cut-off has been recently proposed to identify a more severe phenotype of COVID-19 [16]. In our patients, $C_{R S}$ $<40 \mathrm{~mL} / \mathrm{cmH}_{2} \mathrm{O}$ was independently associated with both prolonged mechanical ventilation (Fig. 1) and mortality. In this regard, we also showed that $55 \%$ of patients had $\mathrm{C}_{\mathrm{RS}}<40 \mathrm{~mL} / \mathrm{cmH}_{2} \mathrm{O}$ at starting of $\mathrm{MV}$, while $57 \%$ of patients experienced $\mathrm{C}_{\mathrm{RS}}<40 \mathrm{~mL} / \mathrm{cmH}_{2} \mathrm{O}$ during the first 5 days. Of note, $\mathrm{C}_{\mathrm{RS}}$ after intubation did not differ between patients who were or not liberated from MV but the percentage of patients with $\mathrm{C}_{\mathrm{RS}}<40 \mathrm{~mL} / \mathrm{cmH}_{2} \mathrm{O}$ within 5 days was significantly different. Although our data do not allow definite conclusions, we can underline that a $\mathrm{C}_{\mathrm{RS}}<40 \mathrm{~mL} / \mathrm{cmH}_{2} \mathrm{O}$ possibly represents a marker of worse ventilatory outcome in COVID-19 patients.

As expected from current knowledge on COVID-19 [17], oxygenation was compromised and a high $\mathrm{FiO}_{2}$ (median value $70 \%$ ) was needed. Further, the lowest $\mathrm{PaO}_{2} / \mathrm{FiO}_{2}$ ratio observed during the first 5 days of $\mathrm{MV}$ was a limiting factor for liberation from mechanical ventilation, as it has been demonstrated in "classical" ARDS patients $[2,18,19]$.

The debate on whether COVID-19 patients have a special form of ARDS is still ongoing [17, 20], but recent papers focused on COVID-19-ARDS pathophysiology report values of oxygenation (P/F ratio) and compliance similar to those of "classical" ARDS [12, 19, 21-23]. The totality of our patients matches oxygenation criteria for 
Table 2 Main results_-ICU ventilation data, complications, and outcomes

\begin{tabular}{|c|c|c|c|c|}
\hline & All patients & $\begin{array}{l}\text { Successful liberation } \\
\text { from MV } 28 d\end{array}$ & $\begin{array}{l}\text { Unsuccessful liberation } \\
\text { from MV } 28 \mathrm{~d}\end{array}$ & \\
\hline Initial ventilatory variables ${ }^{a}$ & $n=240^{a}$ & $n=93^{a}$ & $n=147^{a}$ & $p$ \\
\hline Tidal volume set - mL/kg IBW (IQR) & $7.1(6.5-7.8)$ & $7(6.5-7.9)$ & $7.1(6.4-7.7)$ & 0.932 \\
\hline PEEP set - $\mathrm{cmH}_{2} \mathrm{O}(\mathrm{IQR})$ & $12(10-14)$ & $12(10-14)$ & $12(10-14)$ & 0.466 \\
\hline Pplat - $\mathrm{CmH}_{2} \mathrm{O}(\mathrm{IQR})$ & $25(22-27)$ & $25(22-27)$ & $25(22-28)$ & 0.662 \\
\hline $\mathrm{C}_{\mathrm{RS}}$ in supine position $-\mathrm{mL} / \mathrm{cmH}_{2} \mathrm{O}(\mathrm{IQR})$ & $38(32-47)$ & $39(32-48)$ & $37(31-47)$ & 0.297 \\
\hline $\mathrm{C}_{\mathrm{RS}}$ in supine position $<40 \mathrm{~mL} / \mathrm{cmH}_{2} \mathrm{O}-\mathrm{no}(\%)$ & $132(55 \%)$ & $49(52.7 \%)$ & $83(56.5 \%)$ & 0.567 \\
\hline Mechanical ventilation - first 5 days & $n=391$ & $n=165$ & $n=226$ & $p$ \\
\hline Highest $\mathrm{FiO}_{2}$ set for at least $12 \mathrm{~h}-\%(\mathrm{IQR})$ & $70(60-80)$ & $70(60-80)$ & $75(60-90)$ & $<0.001$ \\
\hline Lowest $\mathrm{PaO}_{2} / \mathrm{FiO}_{2}$ ratio in supine position - (IQR) & $100(76-132)$ & $113(90-145)$ & $94(68-123)$ & $<0.001$ \\
\hline $\mathrm{PaO}_{2} / \mathrm{FiO}_{2}$ class & & & & $<0.001^{b}$ \\
\hline 200-300 - no (\%) & $15(3.8 \%)$ & $6(3.6 \%)$ & $9(4.0 \%)$ & \\
\hline 100-200 - no (\%) & 195 (49.9\%) & $106(64.2 \%)$ & $89(39.4 \%)$ & \\
\hline$<100-$ no $(\%)$ & $181(46.3 \%)$ & $53(32.1 \%)$ & $128(56.6 \%)$ & \\
\hline Lowest static $\mathrm{C}_{\mathrm{RS}}-\mathrm{mL} / \mathrm{cmH}_{2} \mathrm{O}(\mathrm{IQR})$ & $37(30-45)$ & $40(33-47)$ & $35(30-43)$ & $<0.001$ \\
\hline $\mathrm{C}_{\mathrm{RS}}<40 \mathrm{~mL} / \mathrm{cmH}_{2} \mathrm{O}-\mathrm{no}(\%)$ & $225(57.5 \%)$ & $77(46.7 \%)$ & $148(65.5 \%)$ & $<0.001$ \\
\hline ICU stay variables & $n=391$ & $n=165$ & $n=226$ & $p$ \\
\hline Liberation from mechanical ventilation - no (\%) & $208(53.2 \%)$ & $165(100 \%)$ & $43(19 \%)$ & - \\
\hline VFD 28 days $-\mathrm{d}(\mathrm{IQR})$ & $14(9-19)$ & $14(9-19)$ & 0 & - \\
\hline Duration of MV - d (IQR) & $16(10-27)$ & $14(9-19)$ & $20(11-33)$ & $<0.001$ \\
\hline Alive $^{c}(n=250)-\mathrm{d}(\mathrm{IQR})$ & $17(10-30)$ & $14(9-19)$ & $35(29-46.5)$ & $<0.001$ \\
\hline Extubation - no (\%) & $89(22.8 \%)$ & $81(49 \%)$ & $8(3.5 \%)$ & $<0.001$ \\
\hline Tracheotomy - no (\%) & $224(57.3 \%)$ & $83(50.3 \%)$ & $141(62.4 \%)$ & 0.022 \\
\hline Time from first tracheal intubation to tracheotomy - d (IQR) & $9(5-12)$ & $7(4-11)$ & $9(6-13)$ & 0.002 \\
\hline Length of ICU stay - days (IQR) & $20(13-32)$ & $18(14-27)$ & $21(11-38)$ & 0.111 \\
\hline Alive $^{d}(n=250)$ - days (IQR) & $24(15-38)$ & $18(14-27)$ & $42(32-51)$ & $<0.001$ \\
\hline Dead $(n=141)-$ days $(\mathrm{IQR})$ & $15(8-22)$ & 0 & $15(8-22)$ & - \\
\hline Post extubation events ${ }^{c}$ & $n=89$ & $n=81$ & $n=8$ & $p$ \\
\hline Extubation failure within $48 \mathrm{~h}$ - no (\%) & $9(10.1 \%)$ & $5(6.1 \%)$ & $4(50 \%)$ & $<0.001$ \\
\hline Extubation failure over $48 \mathrm{~h}$ - no (\%) & $7(7.9 \%)$ & $3(3.7 \%)$ & $4(50 \%)$ & $<0.001$ \\
\hline NIV/CPAP after extubation - no (\%) & $51(57.3 \%)$ & $43(53 \%)$ & $8(100 \%)$ & 0.017 \\
\hline HFNO after extubation - no (\%) & $5(5.6 \%)$ & $9(11 \%)$ & $1(12.5 \%)$ & 0.980 \\
\hline ICU Complications & $n=391$ & $n=165$ & $n=226$ & $p$ \\
\hline Cardiovascular - no (\%) & $66(16.9 \%)$ & $10(6.1 \%)$ & $56(24.8 \%)$ & $<0.001$ \\
\hline Neurologic - no (\%) & $25(6.4 \%)$ & $7(4.2 \%)$ & $18(8.0 \%)$ & 0.137 \\
\hline Gastroenteric - no (\%) & $20(5.1 \%)$ & $6(3.6 \%)$ & $14(6.2 \%)$ & 0.257 \\
\hline Need for renal replacement therapy - no (\%) & $76(19.4 \%)$ & $13(7.9 \%)$ & $63(27.9 \%)$ & $<0.001$ \\
\hline
\end{tabular}


Table 2 Main results-ICU ventilation data, complications, and outcomes (Continued)

\begin{tabular}{|c|c|c|c|c|}
\hline & All patients & $\begin{array}{l}\text { Successful liberation } \\
\text { from MV } 28 d\end{array}$ & $\begin{array}{l}\text { Unsuccessful liberation } \\
\text { from MV } 28 d\end{array}$ & \\
\hline Early onset VAP - no (\%) & $76(19.4 \%)$ & $29(17.6 \%)$ & $47(20.8 \%)$ & 0.427 \\
\hline Lateonset VAP - no (\%) & $175(44.8 \%)$ & $49(29.7 \%)$ & $126(55.8 \%)$ & $<0.001$ \\
\hline Non-pulmonary infections - no (\%) & $112(28.6 \%)$ & $35(21.2 \%)$ & $77(34.1 \%)$ & 0.005 \\
\hline Still in ICU at the end of observation - no (\%) & $39(9.7 \%)$ & $4(2.4 \%)$ & $35(15.5 \%)$ & $<0.001$ \\
\hline ICU mortality ${ }^{\mathrm{d}}$ - no (\%) & $141(36.1 \%)$ & $0(0 \%)$ & $141(62.4 \%)$ & $<0.001$ \\
\hline
\end{tabular}

Abbreviations: IBW ideal body weight, PEEP positive end-expiratory pressure, $P$ plat plateau pressure, $C_{R S}$ respiratory system compliance measured in a supine position, $\mathrm{FiO}_{2}$ inspired fraction of oxygen, $\mathrm{PaO}_{2}$ arterial oxygen partial pressure, $\mathrm{MV}$ mechanical ventilation, NIV non-invasive ventilation, $C P A P$ continuous positive airway pressure, HFNO high flow nasal oxygen, VFD ventilator-free days, VAP ventilator-associated pneumonia

ancomplete data due to transfer from other ICUs without complete medical records

${ }^{b} p$ value referred to Pearson's chi-square test performed on the contingency table represented 'The percentages of extubation failures and NIV/CPAP/HFNO use after extubation are referred to the total number of the patients extubated

dPatients discharged alive from ICU or still in ICU at the end of observation

ARDS, most of them exhibit impaired lung mechanics (low static compliance) and need treatment with relatively high levels of PEEP (median $12 \mathrm{cmH}_{2} \mathrm{O}$ ) [24] and $\mathrm{FiO}_{2}$ (median $70 \%)$ to warrant adequate oxygenation, all characteristics common to "classical" ARDS [25]. This may explain why most of the risk factors for prolonged mechanical ventilation we have identified are common to those of ARDS due to other causes. Of course, some features of ARDS due to COVID-19 seem to be peculiar and particularly important in determining the outcome. One of the main histological findings in COVID-19 ARDS is a significant endothelial involvement due to viral antigen exposure

Table 3 Fine and Gray's competing-risks analysis

\begin{tabular}{|c|c|c|c|c|c|c|}
\hline \multirow[b]{2}{*}{ Variable } & \multicolumn{3}{|c|}{ Univariate analysis } & \multicolumn{3}{|c|}{ Multivariate analysis } \\
\hline & SHR & $95 \% \mathrm{Cl}$ & $p$ & SHR & $95 \% \mathrm{Cl}$ & $p$ \\
\hline Age & 0.968 & $0.959-0.978$ & $<0.001$ & 0.979 & $0.966-0.992$ & 0.002 \\
\hline Sex $(M)$ & 0.716 & $0.491-1.044$ & 0.083 & - & & \\
\hline $\mathrm{BMl}$ & 0.999 & $0.974-1.024$ & 0.922 & - & & \\
\hline SOFA score at ICU admission & 0.836 & $0.788-0.887$ & $<0.001$ & 0.867 & $0.792-0.949$ & 0.002 \\
\hline SAPS II score & 0.973 & $0.959-0.987$ & $<0.001$ & - & & \\
\hline Hypertension & 0.648 & $0.498-0.843$ & 0.001 & - & & \\
\hline Chronic ischemic heart disease & 0.760 & $0.473-1.221$ & 0.257 & - & & \\
\hline COPD (oxygen therapy/CPAP) & 0.982 & $0.219-4.407$ & 0.981 & - & & \\
\hline Chronic kidney disease (CKD) & 0.692 & $0.303-1.582$ & 0.383 & - & & \\
\hline CKD -dialisys & 0.532 & $0.061-4.640$ & 0.568 & - & & \\
\hline Diabetes & 0.703 & $0.459-1.078$ & 0.106 & - & & \\
\hline Chronic liver disease (MELD > 10) & 0.853 & $0.224-3.239$ & 0.815 & - & & \\
\hline Renal replacement therapy during ICU stay & 0.274 & $0.142-0.531$ & $<0.001$ & 0.381 & $0.220-0.660$ & 0.001 \\
\hline Early onset VAP & 0.844 & $0.600-1.188$ & 0.331 & - & & \\
\hline Late-onset VAP & 0.357 & $0.251-0.510$ & $<0.001$ & 0.283 & $0.197-0.407$ & $<0.001$ \\
\hline Lowest $\mathrm{PaO}_{2} / \mathrm{FiO}_{2}$ within 5 days ${ }^{\mathrm{a}}$ & 1.729 & $1.312-2.280$ & $<0.001$ & 1.631 & $1.316-2.023$ & $<0.001$ \\
\hline $\mathrm{C}_{\mathrm{RS}}<40 \mathrm{~mL} / \mathrm{cmH}_{2} \mathrm{O}$ within 5 days & 0.538 & $0.350-0.826$ & 0.005 & 0.488 & $0.317-0.752$ & 0.001 \\
\hline Cardiovascular complications & 0.253 & $0.175-0.367$ & $<0.001$ & 0.277 & $0.181-0.423$ & $<0.001$ \\
\hline Neurologic complications & 0.550 & $0.236-1.283$ & 0.167 & 0.469 & $0.207-1.061$ & 0.069 \\
\hline Gastrointestinal complications & 0.578 & $0.374-0.894$ & 0.014 & - & & \\
\hline Extrapulmonary infections & 0.533 & $0.326-0.872$ & 0.012 & - & & \\
\hline
\end{tabular}

Notes: Event of interest-liberation from mechanical ventilation. Competing event- death. Observation time: 28 days

Abbreviations: SHR subhazard ratio, BMI body mass index, SAPS simplified acute physiology score, SOFA Sequential Organ Failure Assessment Score, COPD chronic obstructive pulmonary disease, $C K D$ chronic kidney disease, MELD model for end-stage liver disease, $\mathrm{PaO}_{2}$ arterial oxygen partial pressure, FiO $\mathrm{O}_{2}$ inspired fraction of oxygen, $C_{R S}$ respiratory system compliance

${ }^{\mathrm{a}} \mathrm{SHR}$ calculated per 100 points increase of $\mathrm{PaO}_{2} / \mathrm{FiO}_{2}$ ratio 

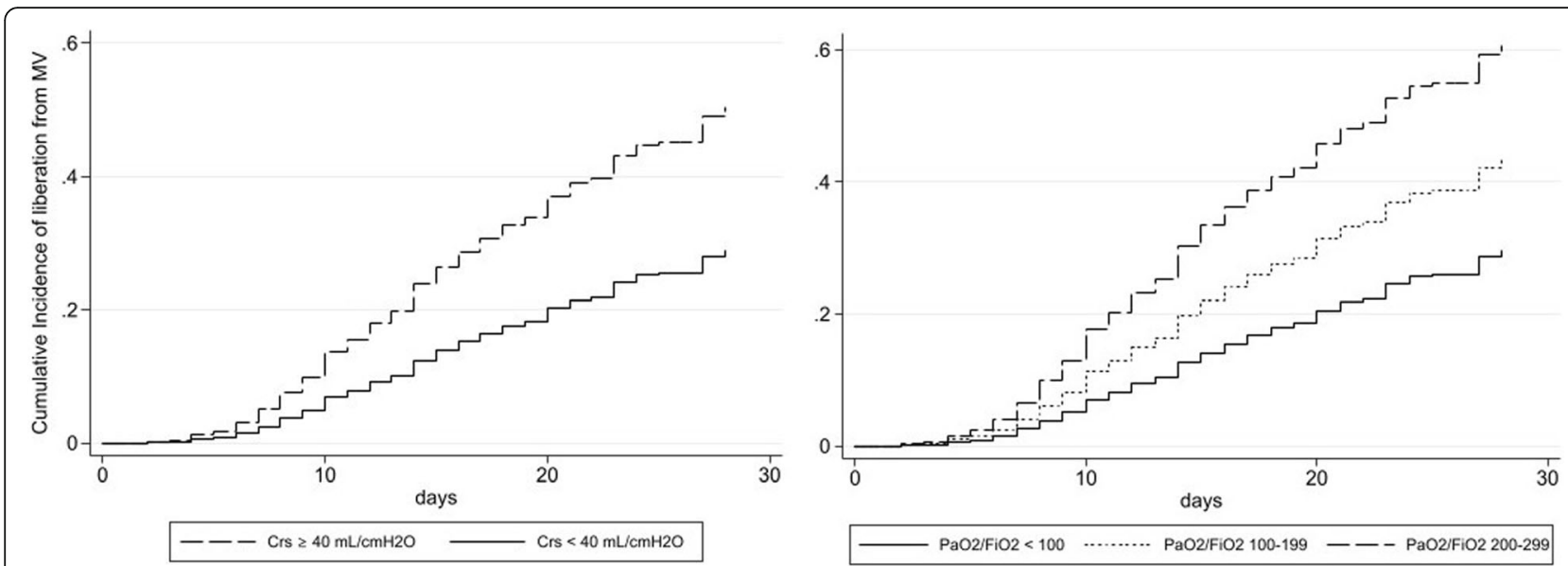

Fig. 1 Cumulative incidence function of liberation from mechanical ventilation on the divided for either static compliance of the respiratory system $\left(\mathrm{C}_{\mathrm{RS}}\right)$ or $\mathrm{PaO}_{2} / \mathrm{FiO}_{2}$ ratio range and adjusted for the other covariates introduced into the multivariate model of the competing-risks regression

Table 4 Multivariate logistic regression analysis, dependant variable death during ICU stay

\begin{tabular}{|c|c|c|c|c|c|c|}
\hline \multirow[b]{2}{*}{ Variable } & \multicolumn{3}{|c|}{ Univariate logistic regression analysis } & \multicolumn{3}{|c|}{ Multivariate logistic regression analysis } \\
\hline & $\mathrm{HR}$ & 95\% C.I. & $p$ & $\mathrm{HR}$ & 95\% C.I. & $p$ \\
\hline Age & 1.067 & $1.029-1.107$ & $<0.001$ & 1.073 & $1.032-1.116$ & $<0.001$ \\
\hline $\operatorname{Sex}(M)$ & 1.120 & $0.735-1.709$ & 0.597 & - & & \\
\hline $\mathrm{BMl}$ & 0.992 & $0.945-1.042$ & 0.761 & - & & \\
\hline SOFA score at ICU admission & 1.215 & $1.095-1.349$ & $<0.001$ & 1.142 & $1.007-1.295$ & 0.038 \\
\hline SAPS II score & 1.042 & $1.022-1.063$ & $<0.001$ & - & & \\
\hline Hypertension & 1.906 & $1.182-3.073$ & 0.008 & - & & \\
\hline Chronic ischemic heart disease & 1.053 & $0.556-1.992$ & 0.875 & - & & \\
\hline COPD (oxygen therapy/CPAP) & 1.454 & $0.060-35.34$ & 0.818 & - & & \\
\hline Chronic kidney disease (CKD) & 1.599 & $0.706-3.617$ & 0.260 & - & & \\
\hline CKD - dialisys & 5.595 & $0.512-61.132$ & 0.158 & - & & \\
\hline Diabetes & 3.583 & $0.793-16.195$ & 0.097 & - & & \\
\hline Chronic liver disease (MELD > 10) & 3.583 & $0.793-16.195$ & 0.097 & - & & \\
\hline Renal replacement therapy during ICU stay & 2.885 & $2.265-3.675$ & $<0.001$ & 2.115 & $1.037-4.310$ & 0.039 \\
\hline Early onset VAP & 1.574 & $0.797-3.108$ & 0.192 & - & & \\
\hline Late-onset VAP & 1.190 & $0.787-1.812$ & 0.410 & - & & \\
\hline Lowest $\mathrm{PaO}_{2} / \mathrm{FiO}_{2}$ within 5 days ${ }^{\mathrm{a}}$ & 0.396 & $0.248-0.631$ & $<0.001$ & 0.383 & $0.239-0.613$ & $<0.001$ \\
\hline $\mathrm{C}_{\mathrm{RS}}<40 \mathrm{~mL} / \mathrm{cmH}_{2} \mathrm{O}$ within 5 days & 2.844 & $2.221-3.642$ & $<0.001$ & 3.193 & $2.525-4.040$ & $<0.001$ \\
\hline Cardiovascular complications & 4.701 & $2.807-7.874$ & $<0.001$ & 4.600 & $2.474-8.554$ & $<0.001$ \\
\hline Neurologic complications & 0.673 & $0.233-1.947$ & 0.465 & - & & \\
\hline Gastrointestinal complications & 0.749 & $0.288-1.952$ & 0.555 & - & & \\
\hline Extrapulmonary infections & 0.877 & $0.488-1.578$ & 0.662 & - & & \\
\hline
\end{tabular}

Notes: AUC of the ROC curve for the multivariate model: 0.818

Abbreviations: $H R$ hazard ratio, C.I. confidence interval, BMI body mass index, SAPS simplified acute physiology score, SOFA Sequential Organ Failure Assessment Score, COPD chronic obstructive pulmonary disease, CKD chronic kidney disease, MELD model for end-stage liver disease, VAP ventilator-associated pneumonia, $\mathrm{PaO}_{2}$ arterial oxygen partial pressure, $\mathrm{FiO}_{2}$ inspired fraction of oxygen, $\mathrm{C}_{\mathrm{RS}}$ respiratory system compliance

${ }^{\mathrm{a}}$ Per 100 points increase of $\mathrm{PaO}_{2} / \mathrm{FiO}_{2}$ ratio 
and cytokine activation that leads to inflammation activation and possible cytokine storm, mostly through the activation of an angiotensin-converting enzyme 2 (ACE2) receptor (expressed in pulmonary endothelial cells, alveolar epithelial type II cells, heart, intestine, and kidney). This histological damage can in part explain the prothrombotic state of COVID-19 patients, and it has been observed that higher D-dimer levels correlate with the severity of the disease and the in-hospital mortality [26]; moreover, very high mortality rates have been observed if high D-dimer levels are combined with low static compliance values [22].

The third category of risk factors for prolonged mechanical ventilation we identified is represented by the complications during ICU stay. Indeed, the survival outcome of mechanically ventilated patients depends not only on baseline characteristics but also on further development of complications. These can be further subdivided into non-pulmonary and pulmonary. Among nonpulmonary complications, the most frequent were acute kidney injury needing renal replacement therapy (19\%) and cardiovascular events (17\%).

Acute kidney injury has already been described in COVID-19 patients; although pathophysiological mechanisms should be further characterized, SARS-CoV-2 seems to affect the kidney directly or indirectly $[27,28]$. The cause of kidney involvement in COVID-19 is likely to be multifactorial: endothelial damage due to virus particles, an ACE2-dependent pathway causing cellular dysfunction, and immune response dysregulation in a hypercoagulability and endotheliitis state are probably the most important contributor to acute kidney injury [29-31]. Patients developing acute kidney injury during ICU stay frequently have comorbidities [32,33], and they usually have less favorable outcome [33] and easily develop complications related to fluid overload [34]; consequently, acute kidney injury is a known risk factor for prolonged mechanical ventilation in critically ill patients, regardless of the underlying disease $[35,36]$.

A high rate of cardiovascular events in COVID-19 patients has already been reported [37-39]; in particular, myocardial injury was described in $7.2 \%$ of patients overall and in $22 \%$ of patients requiring ICU admission [40], a percentage similar to the one recorded in our study (17\%). The pathophysiologic mechanisms are still unclear, the most probably being (a) the direct viral action on the myocardium (i.e., SARS-CoV-2 myocarditis) and (b) the cardiomyopathy caused by cytokine storm (similar to septic cardiomyopathy) [37]. Our data show that cardiovascular complications are a strong predictor not only of mortality, but also of delayed separation from mechanical ventilation.

Among pulmonary complications, infections played a major role. Late-onset ventilator-associated pneumonia (VAP) had a relevant incidence $(44.8 \%$ ) in our patients, considerably higher than that observed in other studies on "classical" ARDS [41]. This result could be explained by the frequent use of immunomodulatory agents as adjunctive therapies [42] or to SARS-CoV-2 infection per se [43] (see supplement Table 1). Although the description of incidence, risk factors, and microbiology of lateonset VAP goes beyond the aims of this study, we believe that this aspect can be of clinical relevance, deserving future studies.

Besides the high incidence of VAP, the high rate of tracheostomy may be related to the length of MV and the relatively high incidence of reintubation. The median duration of invasive ventilation in our COVID-19 patients was 16 days, and this is in line with other recent reports, showing a range of 10-18 days [12, 19, 21, 23]; meanwhile, in "classical" ARDS durations between 6 days for mild and 11 days for severe ARDS have been reported [2]. The reason for this discrepancy has to be further investigated, but we can formulate some hypotheses: first, ARDS patients in the LUNG-SAFE cohort were much more heterogeneous than our cohort of viral pneumonias due to SARS-CoV-2 infection; second, in COVID-19 patient, the involvement of the central nervous system [44], heart [45], and kidney [30] is frequent, thus explaining the longer need for mechanical ventilation and the noticeably long ICU length of stay (median 20 days overall, 24 days in surviving patients) we recorded in our population. Indeed, $57.3 \%$ of the patients underwent tracheostomy within a median of 9 days [5-12] from intubation. Although a precise definition of "early" and "late" tracheostomy is still lacking, our patients can be placed in the upper range of early tracheostomy, according to previous trials $[46,47]$. To date, no guidelines exist on the optimal timing of tracheostomy in COVID-19 patients, although an expert consensus suggests delaying tracheostomy at least 10 days after intubation [48].

This study has some limitations. First, although data collection was prospective, ventilatory treatment and weaning were not standardized among participating centers, thus adding potential confounding factors. Second, for many variables, we asked the participating centers to collect the lowest values in the first 5 days of ICU stay, thus possibly missing valuable data on the precise timecourse of these variables. Third, various experimental COVID-19 therapies were tested in different centers during the conduction of this study (see Table S1, online supplement). In order to control for the center-related effects, clusterization was adopted for statistical analysis. Finally, 22 patients had not completed the observation period for outcome measures, either because they were still in the ICU at the end of the observation period or because they were transferred for logistical reasons to other non-participating ICUs; in any case, censoring before the end of observation was taken into account by competing risks regression analysis.

Even though regional and national healthcare systems were experiencing high levels of stress at the time of data 
collection, no rationing of healthcare resources [49] was in place in any participating center. Accordingly, although organizational issues do not fall within the scope of this paper, we can exclude that organizational issues may have contributed to unfavorable outcomes.

Despite these limitations, to the best of our knowledge, this is the first study to elucidate the risk factors associated with prolonged mechanical ventilation in COVID-19 patients. Our findings may help clinicians predict the risk for prolonged mechanical ventilation. Once patients with multiple risk factors are identified, clinicians should consider the possibility of a high failure rate of life-sustaining interventions and discuss the possible shift towards a palliative approach. Such a decision should be rigorous and comprehensive of all clinical information. However, additional large-scale studies are still warranted to validate our findings.

\section{Conclusions}

Patients with COVID-19 exhibited a high risk of failure from MV liberation at 28 days. Age, SOFA score, $\mathrm{PaO}_{2} /$ $\mathrm{FiO}_{2}$ ratio, $\mathrm{C}_{\mathrm{RS}}$, need for renal replacement therapy, lateonset VAP, and cardiovascular complications were all independent risk factors for prolonged mechanical ventilation.

\section{Supplementary information}

Supplementary information accompanies this paper at https://doi.org/10. 1186/s40560-020-00499-4.

\section{Additional file 1:.}

\section{Abbreviations}

MV: Mechanical ventilation; ICU: Intensive care unit; $C_{R S}$ : Static respiratory system compliance; ECMO: Extracorporeal membrane oxygenation; $\mathrm{ECCO}_{2} \mathrm{R}$ : Extracorporeal $\mathrm{CO}_{2}$ removal; RRT: Renal replacement therapy; SOFA score: Sequential Organ Failure Assessment; SAPS II score: Simplified Acute Physiology Score; VAP: Ventilator-associated pneumonia

\section{Acknowledgements}

ICU-RER COVID-19 Collaboration - list of collaborators (to be indexed and searchable in PubMed)

Maggiore Hospital Carlo Alberto Pizzardi, Bologna, IT: Marco Tartaglione, Valentina Chiarini, Virginia Buldini, Carlo Coniglio, Federico Moro.

Bellaria Hospital, Bologna, IT: Nicola Cilloni, Lorenzo Giuntoli, Angela

Bellocchio, Emanuele Matteo.

Sant'Orsola-Malpighi University Hospital, Bologna, IT: Giacinto Pizzilli,

Antonio Siniscalchi, Chiara Tartivita, Francesco Matteo.

Imola Hospital, Bologna, IT: Annalisa Marchio, Igor Bacchilega.

Infermi Hospital, Rimini, IT: Laura Bernabé, Sonia Guarino, Elena Mosconi.

M.Bufalini Hospital, Cesena, IT: Luca Bissoni, Lorenzo Viola.

Santa Maria Annunziata Hospital, Firenze, IT: Tommaso Meconi, Vittorio Pavoni.

SS. Trinità Hospital, ASL Novara, IT: Aline Pagni, Patrizia Pompa Cleta, Marco Cavagnino.

Bentivoglio Hospital, Bentivoglio, IT: Anna Malfatto, Angelina Adduci, Silvia Pareschi.

University Hospital of Modena, Modena, IT: Gabriele Melegari, Jessica

Maccieri, Elisa Marinangeli.

Azienda Ospedaliera SS. Antonio e Biagio e Cesare Arrigo, Alessandria, IT:

Fabrizio Racca.

University of Ferrara, Azienda Ospedaliero-Universitaria S. Anna, Cona,

Ferrara, IT: Marco Verri, Giulia Falò, Elisabetta Marangoni.
Villa Erbosa Hospital, San Donato Group, Bologna, IT: Francesco Boni. Santa Maria delle Croci Hospital, Ravenna, IT: Giulia Felloni, Federico Domenico Baccarini.

Morgagni-Pierantoni Hospital, Forlì, IT: Marina Terzitta, Stefano Maitan. Radiology Collaborators (to be indexed and searchable into PubMed) Maggiore Hospital Carlo Alberto Pizzardi, Bologna, IT: Michele Imbriani, Paolo Orlandi

Bellaria Hospital, Bologna, IT: Giorgia Dalpiaz

Sant'Orsola-Malpighi University Hospital, Bologna, IT: Rita Golfieri, Federica Ciccarese

Imola Hospital, Bologna, IT: Antonio Poerio

Infermi Hospital, Rimini, IT: Francesco Muratore, Fabio Ferrari

M.Bufalini Hospital, Cesena, IT: Martina Mughetti

SS. Trinità Hospital, ASL Novara, IT: Loredana Franchini, Ersenad Neziri Bentivoglio Hospital, Bentivoglio, IT: Marco Miceli

Santa Maria delle Croci Hospital, Ravenna, IT: Maria Teresa Minguzzi, Lorenzo Mellini

Morgagni-Pierantoni Hospital, Forlì, IT: Sara Piciucchi

\section{Authors' contributions}

$L G, T, S S, G Z, C C, E G$, and CAM conceived and designed the work. EB, AC, FC, FD, MF, EG, GG, CL, MCL, ML, AM, GN, IO, RP, AP, ER, and ST acquired and interpreted the data. MLBR performed the statistical analysis. LG, TT, SS, GZ, and AF drafted the article. DC, CAV, and VMR substantively revised the article. The ICU-RER COVID-19 collaboration was involved in data collection (see the "Acknowledgements" section). All the authors have approved the submitted version and have agreed both to be personally accountable for the authors' own contributions and to ensure that questions related to the accuracy or integrity of any part of the work, even ones in which the author was not personally involved.

Funding

None declared

\section{Availability of data and materials}

The datasets used and/or analyzed during the current study are available from the corresponding author on reasonable request.

\section{Ethics approval and consent to participate}

The study was approved by the Institutional Review Board (IRB) of the study coordinator center (Maggiore Hospital, Bologna, Italy, approval number: 273/ 2020/OSS/AUSLBO) and by each institutional review committee of the participating hospitals. Informed consent was waived for unconscious patients while it was acquired for conscious patients or after liberation from mechanical ventilation. The researchers analyzed anonymized individual data.

Consent for publication

Not applicable.

\section{Competing interests}

The authors have no conflict of interest to declare.

\section{Author details}

${ }^{1}$ Department of Anaesthesia, Intensive Care and Prehospital Emergency, Ospedale Maggiore Carlo Alberto Pizzardi, Bologna, Italy. ${ }^{2}$ Alma Mater Studiorum, Dipartimento di Scienze Mediche e Chirurgiche, Anesthesia and Intensive Care Medicine, Policlinico di Sant'Orsola, Università di Bologna, Bologna, Italy. ${ }^{3}$ Department of Morphology, Surgery and Experimental Medicine, Section of Anaesthesia and Intensive Care University of Ferrara, Azienda Ospedaliero-Universitaria S. Anna, Via Aldo Moro, 8, 44121 Ferrara, Cona, Italy. ${ }^{4}$ Department of Anesthesia and Intensive Care, Santa Maria delle Croci Hospital, Ravenna, Italy. ${ }^{5}$ Cardio-Anesthesiology Unit, Cardio-Thoracic-Vascular Department, S.Orsola Hospital, University of Bologna, Bologna, Italy. ${ }^{6}$ Radiology Department, M.Bufalini Hospital, Cesena, Italy. ${ }^{7}$ Alma Mater University, Department of Clinical, Integrated and Experimental Medicine (DIMES), Statistical Service, S. Orsola-Malpighi Hospital Bologna, Bologna, Italy. ${ }^{8}$ Department of Anaesthesiology, University Hospital of Modena, Via del Pozzo 71, 41100 Modena, Italy. ${ }^{9}$ Anaesthesia and Intensive Care Department, SS. Trinità Hospital, ASL, Novara, Italy.

${ }^{10}$ Translational Medicine Department, Eastern Piedmont University, Novara, Italy. ${ }^{11}$ Anaesthesia and Intensive Care Residency Program - Translational 
Medicine Department, Eastern Piedmont University, Novara, Italy. ${ }^{12}$ Department of Anaesthesia, Intensive Care and Pain Therapy - Imola Hospital, Imola, Italy. ${ }^{13}$ Anaesthesia and Intensive Care Unit, M. Bufalini Hospital, Cesena, Italy. ${ }^{14}$ Division of Anesthesiology, Hospital S. Orsola Malpighi, Alma Mater Studiorum University of Bologna, Bologna, Italy. ${ }^{15}$ Department of Anesthesia and Intensive Care, G.B. Morgagni-Pierantoni Hospital, Forli, Italy. ${ }^{16}$ Department of Anaesthesia and Intensive Care, Azienda Ospedaliera SS. Antonio e Biagio e Cesare Arrigo, Alessandria, Italy.

${ }^{17}$ Department of Anaesthesia and Intensive Care, Infermi Hospital, Rimini, Italy. ${ }^{18}$ Anaesthesia and Intensive Care Unit, Santa Maria Annunziata Hospital, Firenze, Italy. ${ }^{19}$ Anaesthesia and Intensive Care Unit, Bentivoglio Hospital, Bentivoglio, Italy.

Received: 30 July 2020 Accepted: 7 October 2020

Published online: 15 October 2020

\section{References}

1. Grasselli G, Zangrillo A, Zanella A, Antonelli M, Cabrini L, Castelli A, et al. Baseline characteristics and outcomes of 1591 patients infected with SARSCoV-2 admitted to ICUs of the Lombardy region, Italy. JAMA. 2020;323: 1574-81.

2. Bellani G, Laffey JG, Pham T, Fan E, Brochard L, Esteban A, et al. Epidemiology, patterns of care, and mortality for patients with acute respiratory distress syndrome in intensive care units in 50 countries. JAMA. 2016;315:788-800.

3. Richardson S, Hirsch JS, Narasimhan M, Crawford JM, McGinn T, Davidson KW, et al. Presenting characteristics, comorbidities, and outcomes among 5700 patients hospitalized with COVID-19 in the new York City area. JAMA. 2020;10022:E1-8.

4. Auld SC, Caridi-Scheible M, Blum JM, Robichaux C, Kraft C, Jacob JT, et al. ICU and ventilator mortality among critically ill adults with coronavirus disease 2019. Crit Care Med. 2020;48:e799-804.

5. Quah P, Li A, Phua J. Mortality rates of patients with COVID-19 in the intensive care unit: a systematic review of the emerging literature. Crit. Care. NLM (Medline). 2020;24:285.

6. Wang Y, Lu X, Li Y, Chen H, Chen T, Su N, et al. Clinical course and outcomes of 344 intensive care patients with COVID-19. Am J Respir Crit Care Med. 2020;201:1430-4.

7. David P, Garcia W, Fumeaux T, Guerci P, Monika D, Montomoli J, et al. Prognostic factors associated with mortality risk and disease progression in 639 critically ill patients with COVID-19 in Europe: Initial report of the international RISC-19-ICU prospective observational cohort. EClinicalMedicine. 2020;25:100449.

8. Yang X, Yu Y, Xu J, Shu H, Xia J, Liu H, et al. Clinical course and outcomes of critically ill patients with SARS-CoV-2 pneumonia in Wuhan, China: a singlecentered, retrospective, observational study. Lancet Respir Med. 2020;8:475-81.

9. World Health Organization. Clinical management of COVID-19: interim guidance, 27 May 2020.

10. Ranieri VM, Rubenfeld GD, Thompson BT, Ferguson ND, Caldwell E, Fan E, et al. Acute respiratory distress syndrome: the Berlin definition. JAMA. 2012; 307:2526-33.

11. Yehya N, Harhay MO, Curley MAQ, Schoenfeld DA, Reeder RW. Reappraisal of ventilator-free days in critical care research. Am J Respir Crit Care Med. 2019;200:828-36

12. Cummings MJ, Baldwin MR, Abrams D, Jacobson SD, Meyer BJ, Balough EM, et al. Epidemiology, clinical course, and outcomes of critically ill adults with COVID-19 in new York City: a prospective cohort study. Lancet. 2020;395:1763-70.

13. Luo L, Shaver CM, Zhao Z, Koyama T, Calfee CS, Bastarache JA, et al. Clinical predictors of hospital mortality differ between direct and indirect ARDS. Chest. 2017;151:755-63.

14. Sellares J, Ferrer M, Cano E, Loureiro H, Valencia M, Torres A. Predictors of prolonged weaning and survival during ventilator weaning in a respiratory ICU. Intensive Care Med. 2011;37:775-84.

15. Balzer F, Menk M, Ziegler J, Pille C, Wernecke KD, Spies C, et al. Predictors of survival in critically ill patients with acute respiratory distress syndrome (ARDS): an observational study. BMC Anesthesiol. 2016;16:1-8.

16. Gattinoni L, Chiumello D, Rossi S. COVID-19 pneumonia: ARDS or not? Crit Care. 2020;24:154

17. Gattinoni L, Coppola S, Cressoni M, Busana M, Rossi S, Chiumello D. COVID19 does not lead to a "typical" acute respiratory distress syndrome. Am J Respir Crit Care Med. 2020;201:1299-300.
18. Ochiai R. Mechanical ventilation of acute respiratory distress syndrome. J intensive care. J Intensive Care. 2015;3:1-9.

19. Ziehr DR, Alladina J, Petri CR, Maley JH, Moskowitz A, Medoff BD, et al. Respiratory pathophysiology of mechanically ventilated patients with COVID-19: a cohort study. Am J Respir Crit Care Med. 2020;201:1560-4.

20. Fan E, Beitler JR, Brochard L, Calfee CS, Ferguson ND, Slutsky AS, et al. COVID-19-associated acute respiratory distress syndrome: is a different approach to management warranted? Lancet Respir Med. 2020;8:816-21.

21. Schenck EJ, Hoffman K, Goyal P, Choi J, Torres L, Rajwani K, et al. Respiratory mechanics and gas exchange in COVID-19-associated respiratory failure. Ann Am Thorac Soc. 2020;17:1158-61.

22. Grasselli G, Tonetti T, Protti A, Langer T, Girardis M, Bellani G, et al. Pathophysiology of COVID-19-associated acute respiratory distress syndrome: a multicentre prospective observational study. Lancet Respir Med. 2020. https://doi.org/10.1016/S2213-2600(20)30370-2.

23. Ferrando C, Suarez-Sipmann F, Mellado-Artigas R, Hernández M, Gea A, et al. Clinical features, ventilatory management, and outcome of ARDS caused by COVID-19 are similar to other causes of ARDS. Intensive Care Med. 2020. https://doi.org/10.1007/s00134-020-06192-2.

24. Grasso S, Mirabella L, Murgolo F, Di Mussi R, Pisani L, Dalfino L, et al. Effects of positive end-expiratory pressure in "high compliance" severe acute respiratory syndrome coronavirus 2 acute respiratory distress syndrome. Crit Care Med. 2020. https://doi.org/10.1097/CCM.0000000000004640.

25. Spadaro S, Park M, Turrini C, Tunstall T, Thwaites R, Mauri T, et al. Biomarkers for acute respiratory distress syndrome and prospects for personalised medicine. J Inflamm. 2019. https://doi.org/https://doi.org/10.1186/s12950-018-0202-y.

26. Zhang L, Yan X, Fan Q, Liu H, Liu X, Liu Z, et al. D-dimer levels on admission to predict in-hospital mortality in patients with Covid-19. J Thromb Haemost. 2020;18:1324-9.

27. Su H, Yang M, Wan C, Yi LX, Tang F, Zhu HY, et al. Renal histopathological analysis of 26 postmortem findings of patients with COVID-19 in China. Kidney Int. 2020;98:219-27.

28. Cheng $Y$, Luo $R$, Wang $K$, Zhang $M$, Wang Z, Dong $L$, et al. Kidney disease is associated with in-hospital death of patients with COVID-19. Kidney Int. 2020;97:829-38.

29. Varga Z, Flammer AJ, Steiger P, Haberecker M, Andermatt R, Zinkernagel AS, et al. Endothelial cell infection and endotheliitis in COVID-19. Lancet. 2020; 395:1417-8.

30. Ronco C, Reis T, Husain-Syed F. Management of acute kidney injury in patients with COVID-19. Lancet Respir Med. 2020;8:738-42.

31. Gupta A, Madhavan MV, Sehgal K, Nair N, Mahajan S, Sehrawat TS, et al. Extrapulmonary manifestations of COVID-19. Nat. Med. 2020;26:1017-32.

32. Pei G, Zhang Z, Peng J, Liu L, Zhang C, Yu C, et al. Renal involvement and early prognosis in patients with COVID-19 pneumonia. J Am Soc Nephrol. 2020;31:1157-65.

33. Hoste EAJ, Bagshaw SM, Bellomo R, Cely CM, Colman R, Cruz DN, et al. Epidemiology of acute kidney injury in critically ill patients: the multinational AKI-EPI study. Intensive Care Med. 2015;41:1411-23.

34. Zhang L, Chen Z, Diao Y, Yang Y, Fu P. Associations of fluid overload with mortality and kidney recovery in patients with acute kidney injury: a systematic review and meta-analysis. J Crit Care. 2015;30:860.e7-13.

35. Ter Chao C, Hou CC, Wu VC, Lu HM, Wang CY, Chen L, et al. The impact of dialysis-requiring acute kidney injury on long-term prognosis of patients requiring prolonged mechanical ventilation: nationwide population-based study. PLoS One. 2012;7:e50675.

36. Joannidis M, Druml W, Forni LG, Groeneveld ABJ, Honore PM, Hoste E, et al. Prevention of acute kidney injury and protection of renal function in the intensive care unit: update 2017: expert opinion of the working group on prevention, AKI section, European Society of Intensive Care Medicine. Intensive Care Med. 2017:43:730-49.

37. Clerkin KJ, Fried JA, Raikhelkar J, Sayer G, Griffin JM, Masoumi A, et al. COVID-19 and cardiovascular disease. Circulation. 2020;141:1648-55.

38. Guo T, Fan Y, Chen M, Wu X, Zhang L, He T, et al. Cardiovascular implications of fatal outcomes of patients with coronavirus disease 2019 (COVID-19). JAMA Cardiol. 2020;5:811-8.

39. Lodigiani C, lapichino G, Carenzo L, Cecconi M, Ferrazzi P, Sebastian T, et al. Venous and arterial thromboembolic complications in COVID-19 patients admitted to an academic hospital in Milan. Italy Thromb Res. 2020;191:9-14.

40. Wang D, Hu B, Hu C, Zhu F, Liu X, Zhang J, et al. Clinical characteristics of 138 hospitalized patients with 2019 novel coronavirus-infected pneumonia in Wuhan. China JAMA. 2020;323:1061-9. 
41. Forel JM, Voillet F, Pulina D, Gacouin A, Perrin G, Barrau K, et al. Ventilatorassociated pneumonia and ICU mortality in severe ARDS patients ventilated according to a lung-protective strategy. Crit Care. 2012;16:R65.

42. Ritchie Al, Singanayagam A. Immunosuppression for hyperinflammation in COVID-19: a double-edged sword? Lancet. 2020;395:1111.

43. Mathew D, Giles JR, Baxter AE, Greenplate AR, Wu JE, Alanio C, et al. Deep immune profiling of COVID-19 patients reveals patient heterogeneity and distinct immunotypes with implications for therapeutic interventions. bioRxiv. 2020; doi: https://doi.org/10.1101/2020.05.20.106401.

44. Zubair AS, MCAlpine LS, Gardin T, Farhadian S, Kuruvilla DE, Spudich S. Neuropathogenesis and neurologic manifestations of the coronaviruses in the age of coronavirus disease 2019. JAMA Neurol. 2020;77:1018-27.

45. Mai F, Del Pinto R, Ferri C. COVID-19 and cardiovascular diseases. J Cardiol. 2020;76:453-8.

46. Sugerman HJ, Wolfe L, Pasquale MD, Rogers FB, O'Malley KF, Knudson M, et al. Multicenter, randomized, prospective trial of early tracheostomy. J trauma - Inj infect Crit care. Lippincott Williams Wilkins. 1997;43:741-7.

47. Terragni PP, Antonelli M, Fumagalli R, Mangione S, Pasetto A. Early vs late tracheotomy for prevention. Jama. 2010;303:1483-9.

48. McGrath BA, Brenner MJ, Warrillow SJ, Pandian V, Arora A, Cameron TS, et al. Tracheostomy in the COVID-19 era: global and multidisciplinary guidance. Lancet Respir Med. 2020:8:717-25.

49. Emanuel EJ, Persad G, Upshur R, Thome B, Parker M, Glickman A, et al. Fair allocation of scarce medical resources in the time of covid-19. N Engl J Med. 2020;382:2049-55.

\section{Publisher's Note}

Springer Nature remains neutral with regard to jurisdictional claims in published maps and institutional affiliations.

Ready to submit your research? Choose BMC and benefit from:

- fast, convenient online submission

- thorough peer review by experienced researchers in your field

- rapid publication on acceptance

- support for research data, including large and complex data types

- gold Open Access which fosters wider collaboration and increased citations

- maximum visibility for your research: over $100 \mathrm{M}$ website views per year

At $\mathrm{BMC}$, research is always in progress.

Learn more biomedcentral.com/submissions 